This item was submitted to Loughborough's Research Repository by the author.

Items in Figshare are protected by copyright, with all rights reserved, unless otherwise indicated.

\title{
Verification of various modelling techniques for simply-supported piezoelectric actuated thin panels
}

PLEASE CITE THE PUBLISHED VERSION

PUBLISHER

Professional Engineering Publishing / @ IMECHE

VERSION

VoR (Version of Record)

LICENCE

CC BY-NC-ND 4.0

\section{REPOSITORY RECORD}

Aglietti, G.S., Paul Cunningham, and R.S. Langley. 2009. "Verification of Various Modelling Techniques for Simply-supported Piezoelectric Actuated Thin Panels". figshare. https://hdl.handle.net/2134/4871. 
This item was submitted to Loughborough's Institutional Repository (https://dspace.lboro.ac.uk/) by the author and is made available under the following Creative Commons Licence conditions.

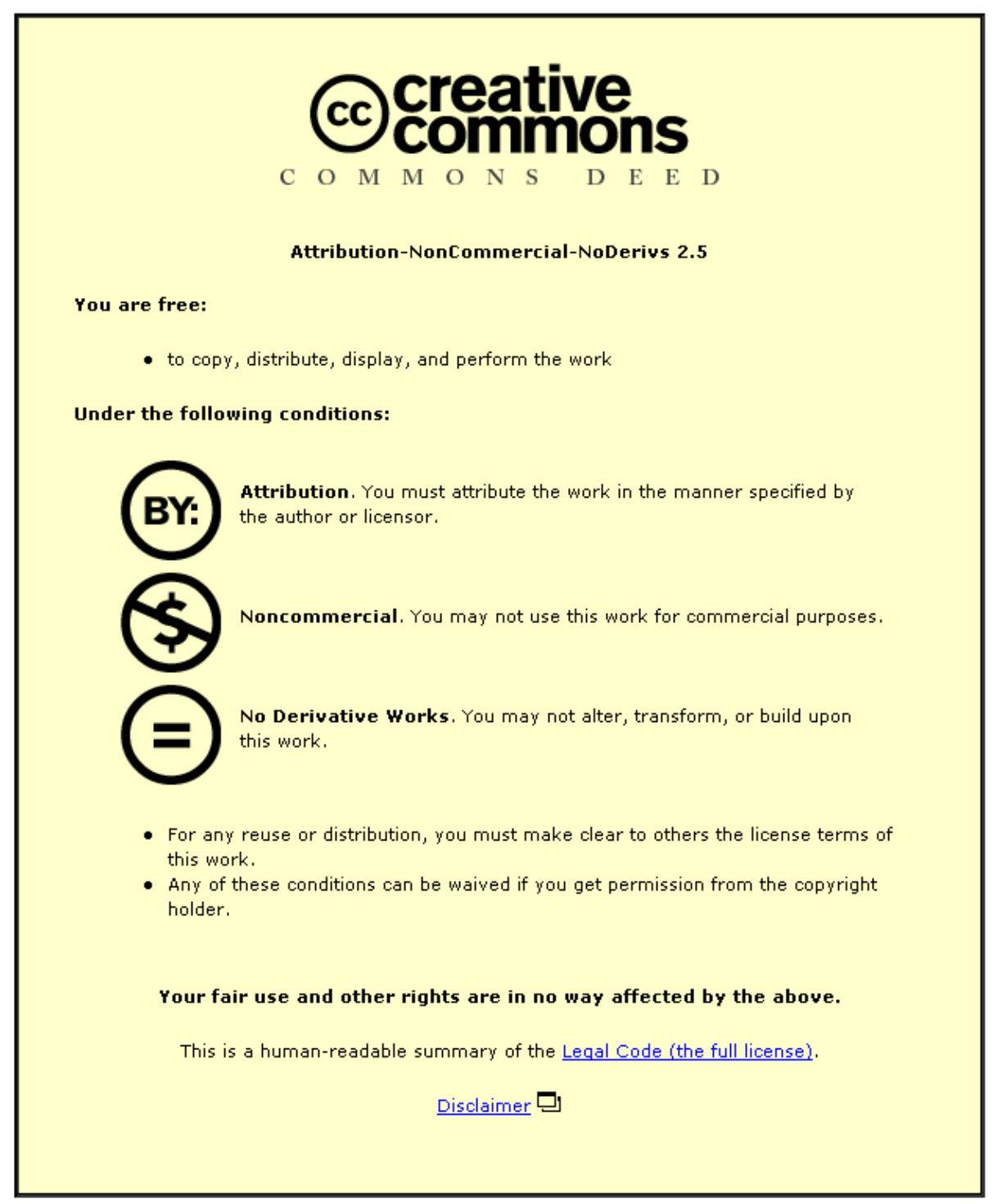

For the full text of this licence, please go to: http://creativecommons.org/licenses/by-nc-nd/2.5/ 


\title{
Verification of various modelling techniques for simply- supported piezoelectric actuated thin panels
}

\author{
G S Aglietti ${ }^{1 *}$, P R Cunningham ${ }^{1}$ and R S Langley $^{2}$ \\ ${ }^{1}$ School of Engineering Sciences, Aeronautics and Astronautics, University of Southampton, Southampton, UK \\ ${ }^{2}$ Department of Engineering, University of Cambridge, Cambridge, UK
}

\begin{abstract}
In this work, three techniques for the mathematical modelling of a piezoelectric actuated thin panel, namely the finite element method, a Lagrange Rayleigh-Ritz method, and a mechanical impedance-based method, are briefly presented. An accurate experimental implementation of a piezoelectric actuated simply-supported panel, whose dynamics have been simulated using the mathematical models, is described in detail. Since the differences between the results produced by the various mathematical models are very small, the accuracy of the experimental set-up is crucial. The results obtained via the numerical simulations are then compared with test results in order to assess the accuracy of the various modelling techniques.
\end{abstract}

Keywords: piezoelectric sensors actuators, simple support, thin panel dynamics, Lagrange RayleighRitz

NOTATI
$\mathrm{d}_{31}, \mathrm{E}_{\mathrm{p}}$
$\mathrm{E}_{\mathrm{pz}}, \mathrm{E}$
$E$
$\mathbf{K}$
$k_{p z}$
$\mathbf{M}$
$M{ }_{\mathrm{x}, \mathrm{y}}$
$\mathrm{q}$
$\mathbf{Q}$
$\mathrm{S}_{\mathrm{m}, \mathrm{n}}$
$t_{\mathrm{pz}}, t_{\mathrm{p}}$
$\mathbf{T}$
$u, v$
$\mathbf{U}$
$v$
$\mathbf{Z}$

piezoelectric constant

Young's modulus of the piezoelectric material and panel, respectively electric field force at the edge of the piezoelectric patch stiffness matrix wave number mass matrix moments at the edges of the piezoelectric patch generalized co-ordinate generalized force mode shapes of the bare panel thickness of the piezoelectric material and panel respectively kinetic energy displacements along the $x$ and $y$ directions potential energy vector containing the voltages at the piezoelectric patches cartesian coordinates mechanical impedance

\footnotetext{
The MS was received on 7 February 2002 and was accepted after revision for publication on 19 A ugust 2002.

* Corresponding author: School of Engineering Sciences, Aeronautics and Astronautics, University of Southampton, Highfield, Southampton SO17 1BJ, UK.
}

$$
\begin{array}{ll}
\phi_{\mathrm{m}, \mathrm{n}} & \begin{array}{l}
\text { modal amplitudes } \\
\text { density of the piezoelectric material and } \\
\rho_{p z}, \rho_{p z}
\end{array} \\
\sigma & \begin{array}{l}
\text { panel, respectively } \\
\text { stress }
\end{array}
\end{array}
$$

\section{INTRODUCTION}

Over the last two decades, many authors have investigated the active control of panel vibrations [1-3]. In order to design active control schemes it is desirable to have simple mathematical models of the plant, i.e. the panel with its sensors and actuators. Although simple, these models have to be accurate enough to represent the essential dynamics of the structure. It is also important to assess the precision with which these models reproduce/simulate the behaviour of the real systems. This specific topic is addressed in this paper, where various mathematical models are compared with each other and with experimental results.

The most suitable type of sensors and actuators for the active control of panel vibrations are piezoelectric patches bonded on the faces of the panel $[4,5]$. The main advantage in the use of piezoelectric patches is the minimum impact on the structure; they are non obtrusive and they do not require a large amount of power or backing structures. Several techniques can be employed to produce mathematical models of this type 
of actively controlled structure [6,7]. Currently the finite element (FE) method is used widely in all areas of structural design and analysis, including the production of models for active control studies. The main advantage of the FE method, compared to other techniques, is the ease with which different types of complex structures can be modelled. However, the drawback of the method is the relatively large size of the mathematical model produced.

For the particular case of panels, several authors have used mechanical impedance-based models [8] as an alternative to the FE method. The interaction between the actuator and the structure is determined by applying equilibrium and compatibility relations at the edges of the patches, so that the actuator's presence is imposed on the underlying structure by a set of line moments/ forces. Such models can be very efficient, although the approximations involved (e.g. to neglect the out-ofplane inertia of the piezoelectric element) can reduce the accuracy of the predicted response.

Energy-based methods have also been used to model the dynamics of actively controlled structures. Hagood et al. [6] present a dynamic model of a beam, with piezoelectric patches, which is derived through the application of Hamilton's principle. Also relevant is the work of Tzou [9] who uses Hamilton's principle and the Kirchhoff-Love thin shell assumptions to derive the equations describing the dynamics of a shell with distributed piezoelectric sensors and actuators. In addition, Banks et al. [10] have used a variational approach to study in detail the interaction between a structural component and an attached piezoelectric patch. The basic principles outlined in references [6], [9] and [10] are incorporated within an energy method developed by Aglietti et al. [11]. The equations governing the dynamics of the system, which includes the panel and the piezoelectric patches working as actuators or sensors, are derived using Lagrange's equations of motion with the vibration mode shapes of the bare panel as the Ritz functions. This approach was validated by comparing results with a detailed FE model. However, the validation of a mathematical model by comparison with another mathematical model does not always guarantee that the models are able to reproduce the real behaviour of the plant.

Several authors have compared response predictions for actively controlled panels with experimental results $[12,13]$, although it is rare to find a detailed description of the experimental arrangement. Since the differences between the results produced by the various mathematical models can be relatively small, the accuracy of the experimental set up is crucial [14]. In this paper the design of an experimental rig to accurately represent a simply-supported actively controlled panel is presented. Various mathematical techniques for modelling the piezoelectric actuated thin panel are briefly described and the results obtained are compared with the experimental data. This enables the sources of discrepancies between the various models to be identified.

\section{MODELS OF THE PIEZOELECTRIC ACTUATED PANEL}

Panels are very common structural elements on board spacecraft, aircraft and other vehicles. For this reason the choice of a panel as the structural element to be modelled and investigated is both analytically convenient and representative of practical applications. It is assumed that the panel is homogeneous and thin enough to be considered in a state of plane stress, which is justified for typical aerospace applications.

A schematic diagram of the arrangement considered in this work is shown in Fig. 1. Two patches of piezoelectric material are bonded onto opposite faces of the panel. The outer electrodes of the patches are electrically connected together and the plate, which is grounded, is used as the other electrode for both patches of the pair. The poling direction of the patches is

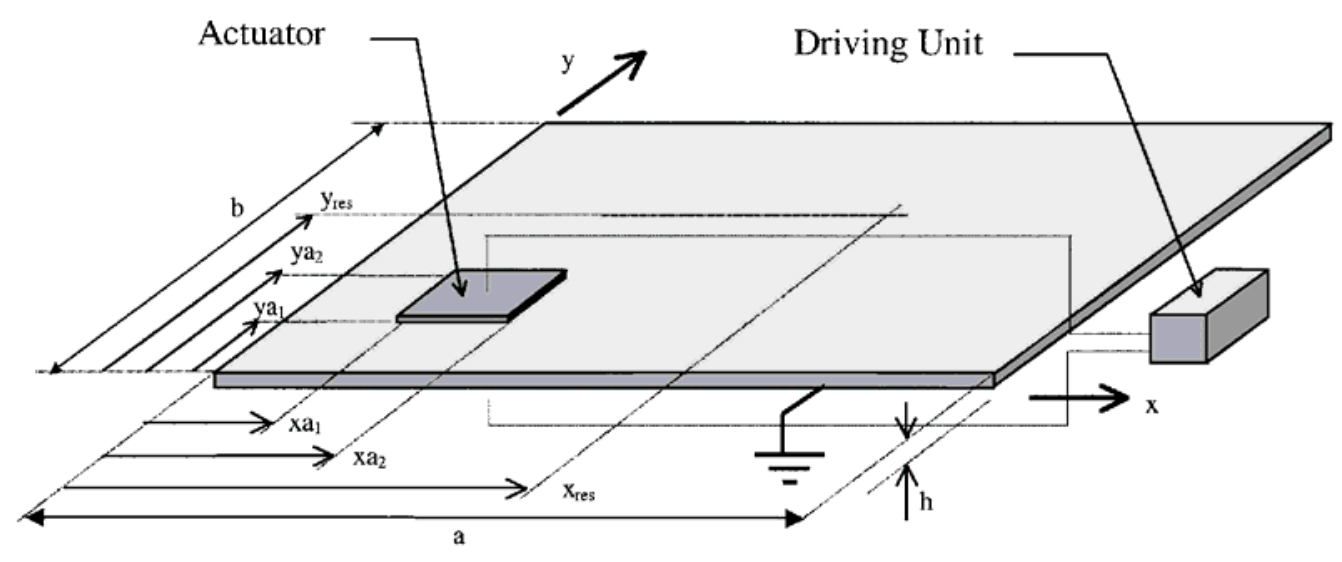

Fig. 1 Piezoelectric actuated panel 


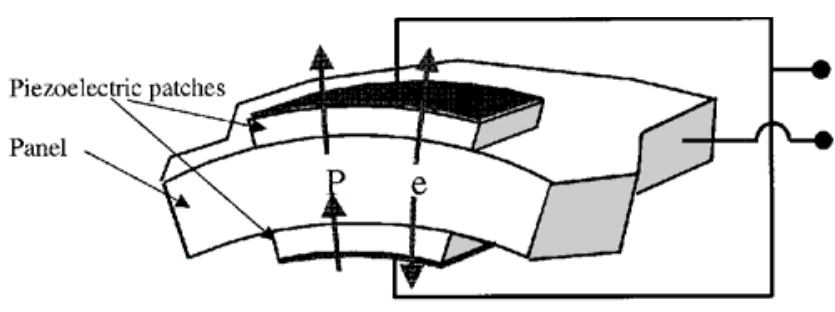

Fig. 2 Cross-section of a piezoelectric patch bonded on the panel during deformation. $\mathrm{P}$ - poling direction of the piezoelectric material; e - induced electric field

directed as shown in Fig. 2. In this configuration, when an external voltage is applied to the patches, one patch will expand and the other contract, thus producing a curvature in the panel. Similarly if the patches are used as sensors, a curvature of the panel (produced by vibration) will induce an electric field in the patches (proportional to the curvature), which can be measured at the electrodes.

\subsection{LRR modelling technique}

A mathematical model of the system can be developed using the Lagrange Rayleigh-Ritz (LRR) procedure described by Aglietti et al. [11]. This method is based on Lagrange's equations of motion which in the general case take the form

$$
\frac{\mathrm{d}}{\mathrm{d} t}\left(\frac{\partial \mathrm{T}}{\partial \dot{\mathrm{q}}_{\mathrm{i}}}\right)-\frac{\partial \mathrm{T}}{\partial \mathrm{q}_{\mathrm{i}}}+\frac{\partial \mathrm{U}}{\partial \mathrm{q}_{\mathrm{i}}}=\mathrm{Q}_{\mathrm{i}}
$$

Here $\mathrm{T}$ and $\mathrm{U}$ are, respectively, the sum of the kinetic and potential energies of the elements composing system (i.e. the panel and piezoelectric patches), and $\mathrm{q}_{\mathrm{i}}$ and $\mathrm{Q}_{\mathrm{i}}$ are, respectively, the $i$ th generalized co-ordinate and generalized force.

The out-of-plane displacement of the panel is written in the form

$$
w(x, y, t)=\sum_{\mathrm{m}=1}^{N m} \sum_{\mathrm{n}=1}^{N n} \mathrm{~S}_{\mathrm{m}, \mathrm{n}}(x, y) \phi_{\mathrm{m}, \mathrm{n}}(t)=\mathrm{s}^{\mathrm{t}} \boldsymbol{\phi}
$$

where $\mathrm{S}_{\mathrm{m}, \mathrm{n}}(x, y)$ represent the mode shapes of the bare panel and $\phi_{\mathrm{m}, \mathrm{n}}$ are the modal amplitudes which form the generalized coordinates for this problem. The kinetic energy of panel, say $\mathrm{T}_{\mathrm{pl}}$ and piezoelectric patches, say $\mathrm{T}_{\mathrm{pz}}$ can be calculated using standard expressions [15], and their final form in terms of the corresponding inertia matrices are

$$
\mathrm{T}_{\mathrm{pl}}=\frac{1}{2} \dot{\boldsymbol{\phi}}^{\mathrm{t}} \mathbf{M}_{\mathrm{pl}} \dot{\boldsymbol{\phi}} \quad, \quad \mathrm{T}_{\mathrm{pz}}=\frac{1}{2} \dot{\boldsymbol{\phi}}^{\mathrm{t}} \mathbf{M}_{\mathrm{pz}} \dot{\boldsymbol{\phi}}
$$

Here $\boldsymbol{\phi}$ is a vector containing the generalized coordinates and full details of the inertia matrices have been given by Aglietti et al. [11]. Similarly, the potential energy due to the elastic deformation of panel and piezoelectric patches can be written as

$$
\mathrm{U}_{\mathrm{pl}}=\frac{1}{2} \boldsymbol{\phi}^{\mathrm{t}} \mathbf{K}_{\mathrm{pl}} \boldsymbol{\phi} \quad, \quad \mathrm{U}_{\mathrm{pz}_{\text {elast }}}=\frac{1}{2} \boldsymbol{\phi}^{\mathrm{t}} \mathbf{K}_{\mathrm{pz}} \mathrm{elast}_{\boldsymbol{\phi}} \boldsymbol{\phi}
$$

Furthermore, due to the piezoelectric effect and the dielectric characteristic of the piezoelectric material, the patch also stores energies that can be called $U_{\text {pzelastelect }}$ and $U_{\text {pzelect}}$, respectively. These energies can be expressed as

$$
\mathrm{U}_{\mathrm{pz}_{\text {elastelect }}}=v^{\mathrm{t}} \mathbf{K}_{\mathrm{pz}_{\text {elastelect }}} \boldsymbol{\phi}, \quad \mathrm{U}_{\mathrm{pz}_{\text {elect }}}=\frac{1}{2} v^{\mathrm{t}} \mathbf{K}_{\mathrm{pz}_{\text {elect }}} v
$$

where $v_{1}$ is the voltage in the $i$ th patch. Again, full details of the various terms that appear in equations (4) and (5) have been given by Aglietti et al. [11].

It is a straightforward task to apply the Lagrange's equation to yield

$$
\begin{aligned}
& \left(\mathbf{M}_{\mathrm{pl}}+\mathbf{M}_{\mathrm{pz}}\right) \ddot{\boldsymbol{\phi}}+\left(\mathbf{K}_{\mathrm{pl}}+\mathbf{K}_{\mathrm{pz}_{\text {elast }}}\right) \boldsymbol{\phi}+\mathbf{K}_{\mathrm{pz}_{\text {elastelect }}}^{\mathrm{t}} v \\
& \quad=\mathbf{Q}
\end{aligned}
$$

When all patches act as actuators, their voltages $v_{\mathrm{i}}$ are externally driven (known) and so the displacement at any location on the panel can be calculated solving equation (6) and substituting the modal displacements in equation (2). If the patches are used as sensors, the voltages at the patches can be considered as degrees of freedom, and therefore the differentiation of the energies with respect to these voltages (according to the Lagrange's equations of motion) produces a further equation

$$
\mathbf{K}_{\mathrm{pz}_{\text {elastelect }}} \phi+\mathbf{K}_{\mathrm{pz}_{\text {elect }}} v=0
$$

which can be used to calculate the voltages as a function of the modal coordinates. This expression can then be substituted into equation (6) to produce a complete set of equations in the unknown $\phi$. Once the modal displacements have been calculated equation (7) can be used to calculate the voltages at the sensors.

\subsection{Mechanical impedance modelling technique}

As an alternative approximate modelling approach, the effect of using a piezoelectric patch as an actuator can be reproduced by using line moments which are applied along the edges of the patch. The main points of this modelling technique are briefly reported in what follows, and full details are given by Zhou et al. [7].

The equations of motion of the piezoelectric patch, along the $x$ and $y$ axes, are taken as

$$
\rho_{p z} \frac{\partial^{2} u}{\partial t^{2}}=\mathrm{E}_{\mathrm{pz}} \frac{\partial^{2} u}{\partial x^{2}} \quad, \quad \rho_{p z} \frac{\partial^{2} v}{\partial t^{2}}=\mathrm{E}_{\mathrm{pz}} \frac{\partial^{2} v}{\partial y^{2}}
$$

where $u$ and $v$ are the dynamic displacements along the $x$ 


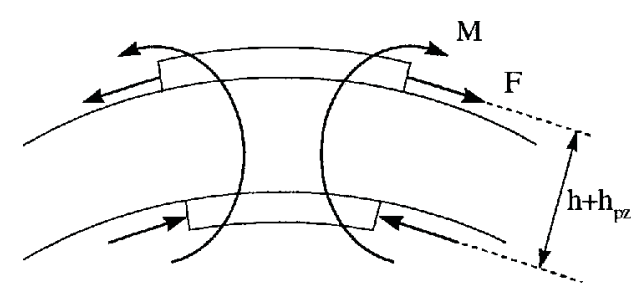

Fig. 3 Forces and line moments produced by the piezoelectric patch

and $y$ directions, and $\rho_{p z}$ and $\mathrm{E}_{\mathrm{pz}}$ are the density and Young's modulus of the piezoelectric material. These equations have the solution

$$
u=A \sin \left(k_{p z} x\right) \mathrm{e}^{j \omega t} \quad, \quad v=B \sin \left(k_{p z} y\right) \mathrm{e}^{j \omega t}
$$

where $k_{p z}$ is the wave number.

Now the constitutive relationships of the piezoelectric material have the form

$$
\begin{aligned}
& \frac{\partial u}{\partial x}=\frac{\sigma_{x}}{\mathrm{E}_{\mathrm{pz}}}-\frac{v_{p z} \sigma_{y}}{\mathrm{E}_{\mathrm{pz}}}+\mathrm{d}_{31} E \\
& \frac{\partial v}{\partial x}=\frac{\sigma_{y}}{\mathrm{E}_{\mathrm{pz}}}-\frac{v_{p z} \sigma_{x}}{\mathrm{E}_{\mathrm{pz}}}+\mathrm{d}_{32} E
\end{aligned}
$$

where $E$ is the electric field applied, and $\mathrm{d}$ is the piezoelectric constant. The stresses in equations $(10 \mathrm{a}, \mathrm{b})$ can be written in terms of forces at the edge of the patch, which are

$$
\mathrm{F}_{y}=\mathbf{Z}_{y y} \dot{v}+\mathbf{Z}_{y x} \dot{u} \quad, \quad \mathrm{~F}_{x}=\mathbf{Z}_{x x} \dot{u}+\mathbf{Z}_{x y} \dot{v}
$$

Here $\mathbf{Z}_{x x} \mathbf{Z}_{y y}$ and $\mathbf{Z}_{x y}$ are the mechanical impedance of the host structure at the edges of the patch. Equations (9)-(11) enable $A$ and $B$ to be expressed in terms of the applied electrical potential $E$, and thus the forces $F_{\mathrm{x}}$ and $F_{\mathrm{y}}$ can also be expressed in terms of this potential. Now the moments at the edge of the patch can be written in the form

$$
\mathbf{M}_{\mathrm{x}, \mathrm{y}}=F_{\mathrm{x}, \mathrm{y}}\left(t_{\mathrm{p}}+t_{\mathrm{pz}}\right)
$$

as illustrated in Fig. 3, and the equation of motion of the panel can be expressed as:

$$
\sum_{\mathrm{m}=1}^{N m} \sum_{\mathrm{n}=1}^{N n}\left(\rho h \omega_{\mathrm{m}, \mathrm{n}}^{2} w_{\mathrm{m}, \mathrm{n}}+\rho h \ddot{w}_{\mathrm{m}, \mathrm{n}}\right)=\frac{\partial \mathbf{M}_{\mathrm{x}}}{\partial_{x}}+\frac{\partial \mathbf{M}_{\mathrm{y}}}{\partial_{y}}
$$

where $\omega_{\mathrm{m}, \mathrm{n}}$ is the resonance frequency of the $\mathrm{m}, \mathrm{n}$ mode, $w_{\mathrm{m}, \mathrm{n}}$ its out-of-plane displacement and $\mathbf{M}_{\mathrm{x}}$ and $\mathbf{M}_{\mathrm{y}}$ can be obtained from (12) and (11).

Equation (13) allows the response of the panel to be computed for an applied input $E$. The mechanical impedance (MI) model has been implemented using MATLAB, and the results are compared with those obtained with the LRR technique, the FE method and the experimental results obtained from the test rig described in the next section.

\subsection{Experimental rig}

A test rig of the simply-supported panel described in section 2 was built in order to verify the results produced by the mathematical models. One of the main problem areas was the realization of the simple supports along the edge of the panel. This problem, addressed by Aglietti and Cunningham [14], was solved by suspending the panel horizontally using shims, as shown in Fig. 4. This set-up produces a negligible rotational stiffness along the edge of the panel, due to the high bending flexibility of the shim. At the same time the high in-plane stiffness of the shim, which is clamped along its upper and lower edges, restrains the out-of-plane (vertical) movements of the edge of the panel. Figure 5 shows the implementation of this type of simple support. The panel details are given in Table 1. The distance between the upper and lower clamped edges of the shim is $40 \mathrm{~mm}$, the thickness of the shims is $0.052 \mathrm{~mm}$, and the panel is bonded along the middle of the shim. The corner pieces (L-section segments of steel beam) onto which the shims are constrained are then bolted to a rigid frame. Another advantage of this type of support is that the out-of-plane flexibility of the shims allows expansion or contraction of the panel (due to temperature changes) without inducing large in-plane prestresses in the panel.

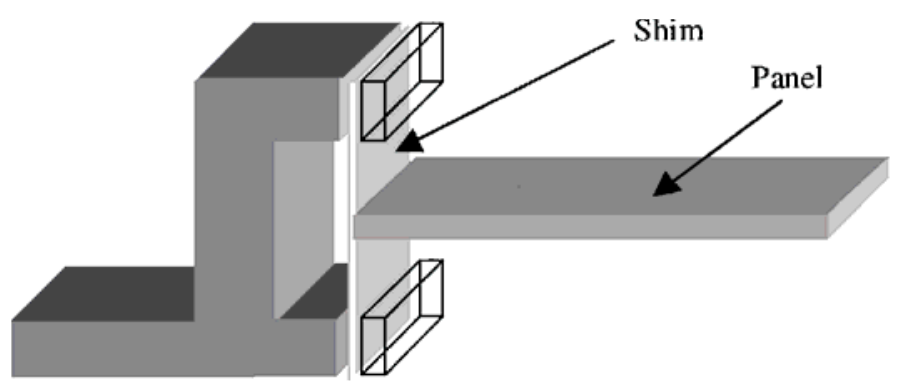

Fig. 4 Schematic of the shim suspension along the edge of the panel 


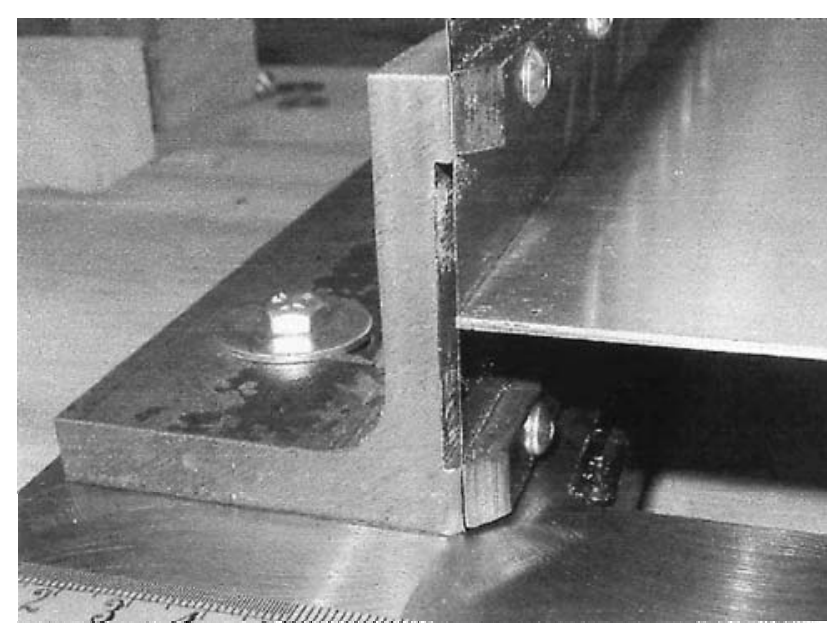

Fig. 5 Experimental implementation of the simple support based on shims

Table 1 Dimensions and material properties

\begin{tabular}{lll}
\hline Panel & $a=304.3 \mathrm{~mm}$ & $\mathrm{E}=71 \mathrm{e} 9 \mathrm{~Pa}$ \\
& $b=203.2 \mathrm{~mm}$ & $\rho=2705 \mathrm{~kg} / \mathrm{m}^{3}$ \\
& $h=1.453 \mathrm{~mm}$ & $v=0.33$ \\
& & $\eta=0.001^{*}$ \\
Actuator/sensor & $x a_{1}=50.8 \mathrm{~mm}$ & $\mathrm{E}=63 \mathrm{e} 9 \mathrm{~Pa}$ \\
& $x a_{2}=101.6 \mathrm{~mm}$ & $\rho=7650 \mathrm{~kg} / \mathrm{m}^{3}$ \\
& $y a_{1}=25.4 \mathrm{~mm}$ & $v=0.3$ \\
& $y a_{2}=76.2 \mathrm{~mm}$ & $d=1.66 \mathrm{e}-10 \mathrm{~m} / \mathrm{V}$ \\
& $h_{\mathrm{pz}}=0.19 \mathrm{~mm}$ & $\varepsilon=1700 \varepsilon^{\circ}$ \\
\hline
\end{tabular}

$* \eta=0$ for the Lagrange/FE model comparison

The corner pieces (L-sections) which support the shims were bolted onto a $13 \mathrm{~mm}$ thick steel plate, with a rectangular cut-out machined in the centre of the plate to the dimensions of the aluminium alloy panel. This cut out is necessary in order to avoid the strong fluidstructure interaction that otherwise would be produced in the gap (cavity) between the aluminium panel and the steel plate. The four L-section steel beams were machined with a channel to produce a gap behind the shims, as shown in Figs 4 and 5. Four steel $\mathrm{U}$-section beams were welded to the bottom of the plate to produce a box-like structure and thus increase the support stiffness.

The aluminium panel was bonded to the shims using epoxy adhesive. The panel was supported while the two long side shims were bonded first. Packing pieces were located behind the shims to prevent movement of the shims during the bonding process. Once the epoxy had cured, the short side shims were bonded to form the complete arrangement. The piezoelectric patches were bonded to the panel using epoxy. The complete test rig, which is shown in Fig. 6, was suspended in a frame using four tension springs to provide freely-supported boundary conditions to the supporting structure.

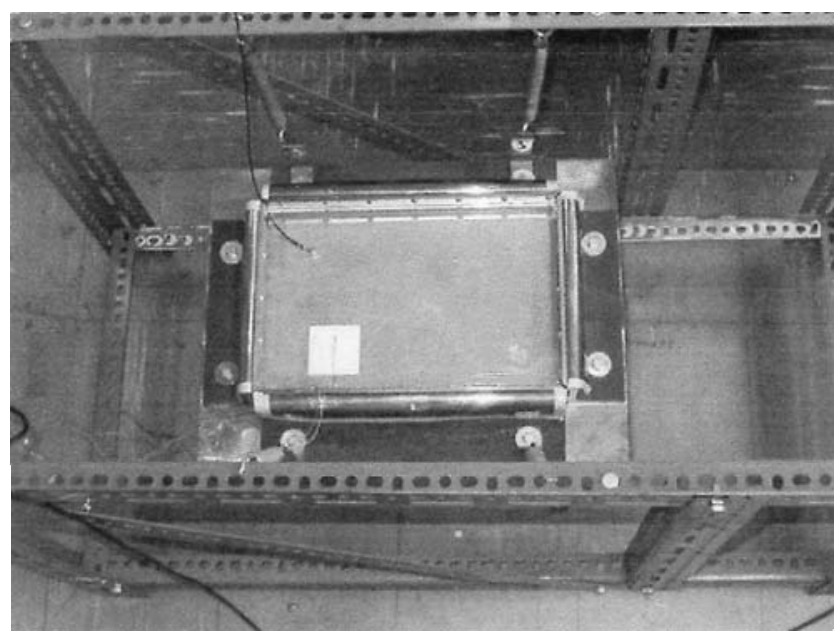

Fig. 6 Test rig supporting frame

\subsection{FE Model}

\subsubsection{Panel with piezoelectric patches}

A FE model of the assembly was constructed using the commercial software package ANSYS (Swanson Analysis Systems Inc.). The model, which is shown in Fig. 7, was composed of 8-node layered shell elements (Shell91), with the areas of the piezoelectric patches modelled using three layers (piezoelectric material upper patch/aluminium/piezoelectric material lower patch). For the rest of the panel a single layer of aluminium was used. Figure 8 shows the multi-layer configuration used to model a piezoelectric patch. The convergence of the FE model shown in Fig. 7 was checked by comparing the results with those produced by a more refined FE model, which is shown in Fig. 9. The decrease in the natural frequencies produced by increasing the number of elements was negligible, thus confirming that the original model was accurate enough for this purpose. The driving force was produced by the contraction/expansion of the piezoelectric patch when the input voltage was applied. In the FE model this effect was produced by applying moments along the edges of the piezoelectric patch. This was done in order to keep the FE model as simple as possible without adding further degrees of freedom such as voltages or temperatures to produce shrinking/expansion of the patches. The moment applied to the edge of the patch is given by

$$
\begin{aligned}
M= & \frac{t_{p}^{2} E_{p} b_{p}\left[1+\left(\frac{t_{p z}}{t_{p}}\right)\right]}{6+\left[\frac{\left(E_{p} b_{p} t_{p}\right)}{\left(E_{p z} b_{p z} t_{p z}\right)}\right]+12\left(\frac{t_{p z}}{t_{p}}\right)+8\left(\frac{t_{p z}}{t_{p}}\right)^{2}} \\
& \times\left(\frac{d_{3,1} V}{t_{p z}}\right)
\end{aligned}
$$

Details concerning this method, which was originally 


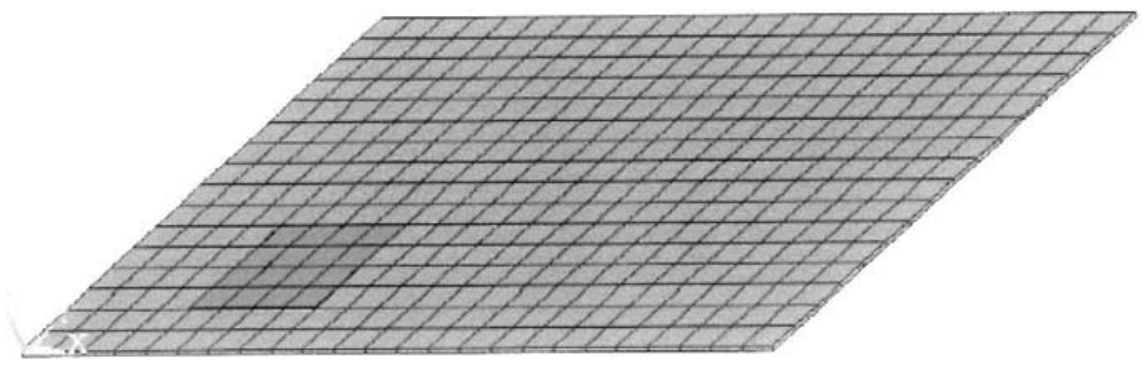

Fig. 7 FE model of the piezoelectric actuated panel

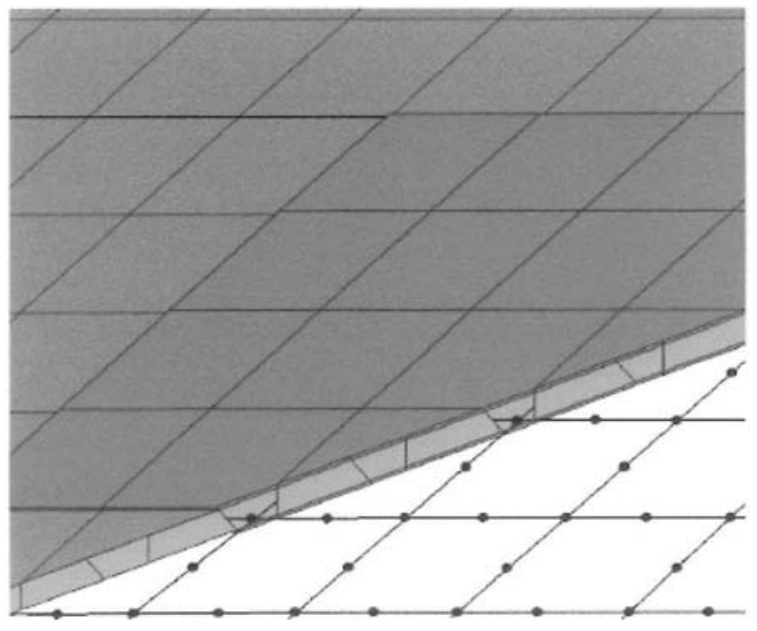

Fig. 8 Cross sectional view of the FE model in the patch area

developed and tested for beams, are given by Brennan et al. [18].

\subsubsection{Complete rig}

The FE model of the whole assembly which was comprised of the panel and supporting structure, is shown in Fig. 10. The L-section beams which support the shims and the metal strips clamping the shims have been modelled using solid elements (with six degrees of freedom per node). The supporting plate and the U-channels welded underneath were modelled using shell elements. The L-sections were coupled to the frame by merging the nodes in the areas where the connecting bolts between these elements were located.

The modes of vibration and the associated natural frequencies were calculated with the whole assembly freely supported, and the non-zero results of this analysis are reported in the fourth and fifth column of Table 2. The addition of a lumped mass ( $2 \mathrm{~g}$ ) to the model, representing an accelerometer positioned on the panel at $x=50.8 \mathrm{~mm}$ and $y=152.4 \mathrm{~mm}$, produces a small decrease in the natural frequencies of vibration (fourth column of Table 2).

\section{TESTS AND DISCUSSION OF RESULTS}

\subsection{Piezoelectric actuated panel}

Before testing, the size and material properties of the aluminium panel were experimentally verified in order to confirm the input data to be used in the mathematical models. The density was verified using a precision scale, after which the Young's modulus was verified by comparing the measured resonant response frequency of the freely supported panel with the calculated natural frequencies of vibration obtained using a FE model. The value of the Young's modulus was also independently validated using a three point static bending test. For the piezoelectric patches, size and density were verified experimentally.

A series of tests was carried out using the experimental rig, exciting the piezoelectric patches with a

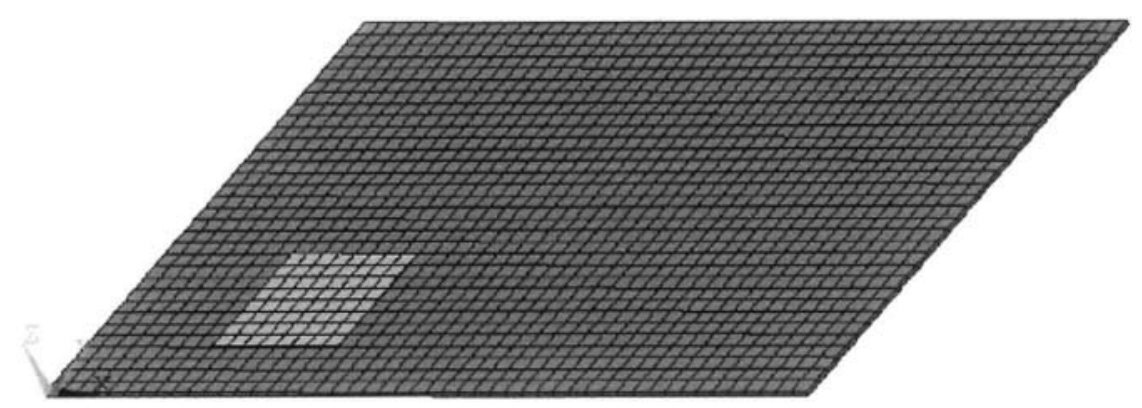

Fig. 9 Refined FE model of the piezoelectric actuated panel 


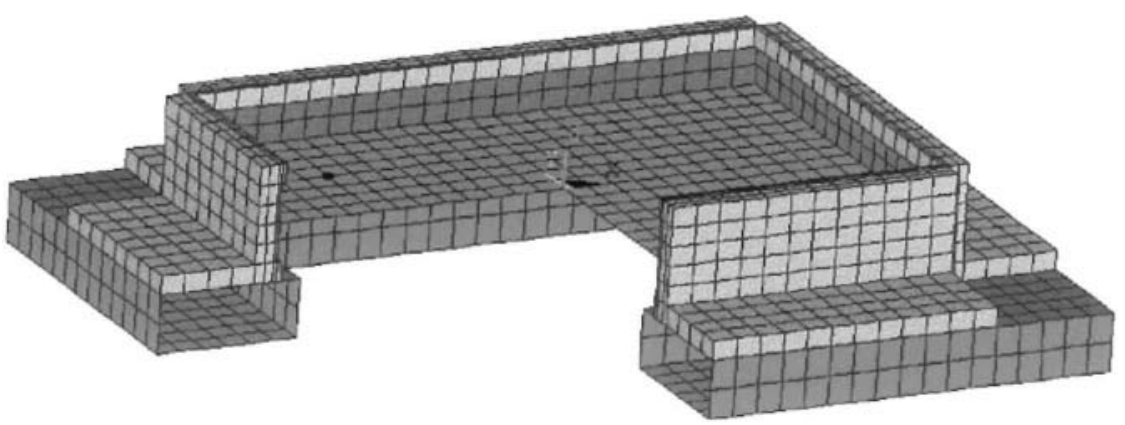

Fig. 10 FE model of the panel suspended by the shims assembled on the frame

Table 2 Comparison of the natural frequencies of vibration between the various modelling techniques and experimental results for the simply supported thin panel

\begin{tabular}{lllllll}
\hline Mode & $\begin{array}{l}\text { Exp. } \\
\text { bare panel }\end{array}$ & $\begin{array}{l}\text { Exp. panel } \\
\text { with patches }\end{array}$ & $\begin{array}{l}\text { FEM panel with } \\
\text { patches and acceler. }\end{array}$ & $\begin{array}{l}\text { FEM panel } \\
\text { with patches }\end{array}$ & $\begin{array}{l}\text { MIM panel } \\
\text { with patches }\end{array}$ & $\begin{array}{l}\text { LRRM panel } \\
\text { with patches }\end{array}$ \\
\hline $\mathbf{1 , 1}$ & 125.3 & 125.0 & 125.3 & 125.5 & 126.0 & 125.2 \\
$\mathbf{2 , 1}$ & 240.5 & 238.5 & 237.7 & 239.0 & 245.0 & 239.4 \\
$\mathbf{1 , 2}$ & 383.9 & 383.0 & 383.6 & 385.3 & 391.5 & 385.0 \\
$\mathbf{3 , 1}$ & 431.5 & 432.5 & 430.9 & 434.3 & 438.0 & 435.1 \\
$\mathbf{2 , 2}$ & 499.0 & 487.5 & 486.3 & 491.0 & 515.0 & 497.3 \\
\hline
\end{tabular}

sinusoidal signal of $2 \mathrm{~V}$ (peak to peak) over the frequency range $50-500 \mathrm{~Hz}$. The lowest limit of the frequency range was imposed to omit the rigid body motion of the system. The upper limit was fixed to avoid exciting the flexible modes of the supporting frame. The lowest natural frequency of the frame was at $550 \mathrm{~Hz}$ and the shims have a natural frequency of $630 \mathrm{~Hz}$. Preliminary calculations indicated that there should be five panel resonances in the selected frequency range, which are adequate for the purpose of the present work. The response of the panel was retrieved using a B\&K accelerometer $(2 \mathrm{~g})$ positioned at $x=50.8 \mathrm{~mm}$ and $y=152.4 \mathrm{~mm}$.

The transfer function between applied voltage and output acceleration is plotted in Fig. 11, and the experimental resonant frequencies are reported in the third column of Table 2. The scatter of experimental results in Fig. 11, at low frequency, was essentially due to electrical noise and it does not affect the issues discussed in this paper. Furthermore, at low frequency, the rigid body modes of the experimental frame suspended by the springs would also affect the results, thus invalidating the comparison with the ideal models (MI and LRR), which did not include the frame and its suspensions.

From the frequency response functions in Fig. 11 it is clear that all theoretical models agree very well with the experimental results. However, a closer inspection shows that the MI model, in the version implemented in this work, does not reproduce the response of the panel as accurately as the other methods. In particular, from Table 2 the resonant frequencies calculated using the MI model are consistently higher than those obtained using the other methods. All three models (namely FE, MI, LRR) whose results are reported in the last three columns of Table 2 do not include the mass of the accelerometer in order to allow a proper comparison between the three models. The comparison between these models and the experimental results is somewhat biased by the presence of the accelerometer in the experimental model. However, the comparison of results between the two FE models (with and without accelerometer) shows that the effect of the accelerometer is very small indeed and does not fully account for the higher frequencies predicted by the MI model with respect to the other methods and the experimental results.

The MI model effectively assumes that the piezoelectric patches are connected to the panel only along the patch edges, and compatibility between the panel and the patch is therefore not imposed in the patch interior. This has the effect of omitting the out-of-plane inertia of the patch, and this explains why the natural frequencies predicted by the MI model are higher than both the other theoretical predictions and experimental results. In fact, the MI model predicts that adding the patch to a panel should raise the natural frequencies due to the increase in stiffness, whereas the FE method and the LRR model predict that the natural frequencies will be lower because of the predominant effect of the mass of the patch; the latter prediction is in agreement with the experimental results. The natural frequencies of vibration of the panel with and without the patches are reported in the second and third columns of Table 2. As 


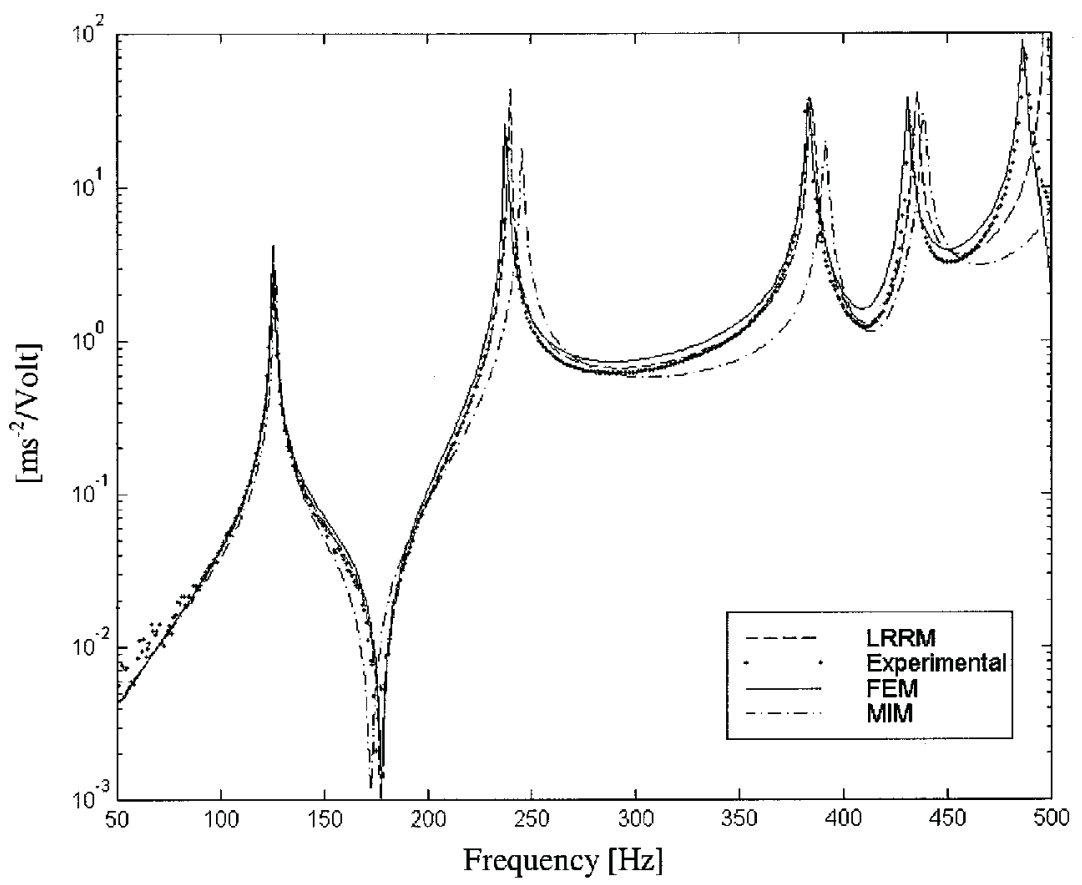

Fig. 11 Transfer functions between voltage applied at the piezoelectric patches and acceleration in $(x=$ $50.8 \mathrm{~mm} y=152.4 \mathrm{~mm}$ )

the thickness (and hence the mass and stiffness) of the patch is reduced the MI model and LRR model agree more closely, as shown in (Fig. 12).

The LRR model agrees very well with the experimental results, and this is particularly relevant since the model only considers the first six by six mode shapes (36 degrees of freedom) and still yields results of accuracy comparable with the FE model, which used approximately 3500 degrees of freedom. In addition the FE model results agree very well with the experimental data, and this confirms that the method used to simulate the effect of the patches with line moments along the edges is accurate.

\subsection{Piezoelectric patches working as sensor}

In many applications the piezoelectric patches are used as sensors, and therefore a new set of tests was performed in order to verify the capability of the mathematical models to simulate this situation. The panel was forced using an Endevco impact hammer with an Isotron force transducer. The panel was tapped at the location $x=254 \mathrm{~mm}$ and $y=152.4 \mathrm{~mm}$, and the accelerometer was retained at the position $x=$ $50.8 \mathrm{~mm}$ and $y=152.4 \mathrm{~mm}$. The voltage produced at the electrodes of the piezoelectric patch was acquired using a Signal Processing Ltd four-channel data acquisition suite [16] connected to a personal computer which operated using MATLAB software [17]. A lumped mass of $2 \mathrm{~g}$ was included in the LRR model to represent the mass of the accelerometer. The force and piezoelectric patch data were post-processed using the MATLAB Spectrum function, and a rectangular window was used since the acceleration signal decayed significantly within the three second data window.

The transfer function produced using the LRR model and the FE model are compared with the experimental results in Fig. 13. In the FE model the voltage produced at the electrodes of the patch was calculated from the difference of the slope (rotations of the nodes) at the opposite edges of the patch. The rotation of the nodes along each edge of the patch were averaged, and then the average along each line of nodes was subtracted from the average of the opposite line. This method, discussed in [18] for piezoelectric patches bonded on beams is extrapolated here and used successfully for patches bonded on panels.

The voltage at the electrodes of the patch in the FE model can also be obtained from the average strain in the patch multiplied by the piezoelectric constant. This method was also tested and yielded identical results.

Overall the FE prediction agrees very well with the experimental results apart from a slight discrepancy at low frequencies. A possible cause for this mismatch is the fact that rigid body modes of the frame could affect the FE results. The LRR model agrees with the experimental results across the whole frequency range investigated. 


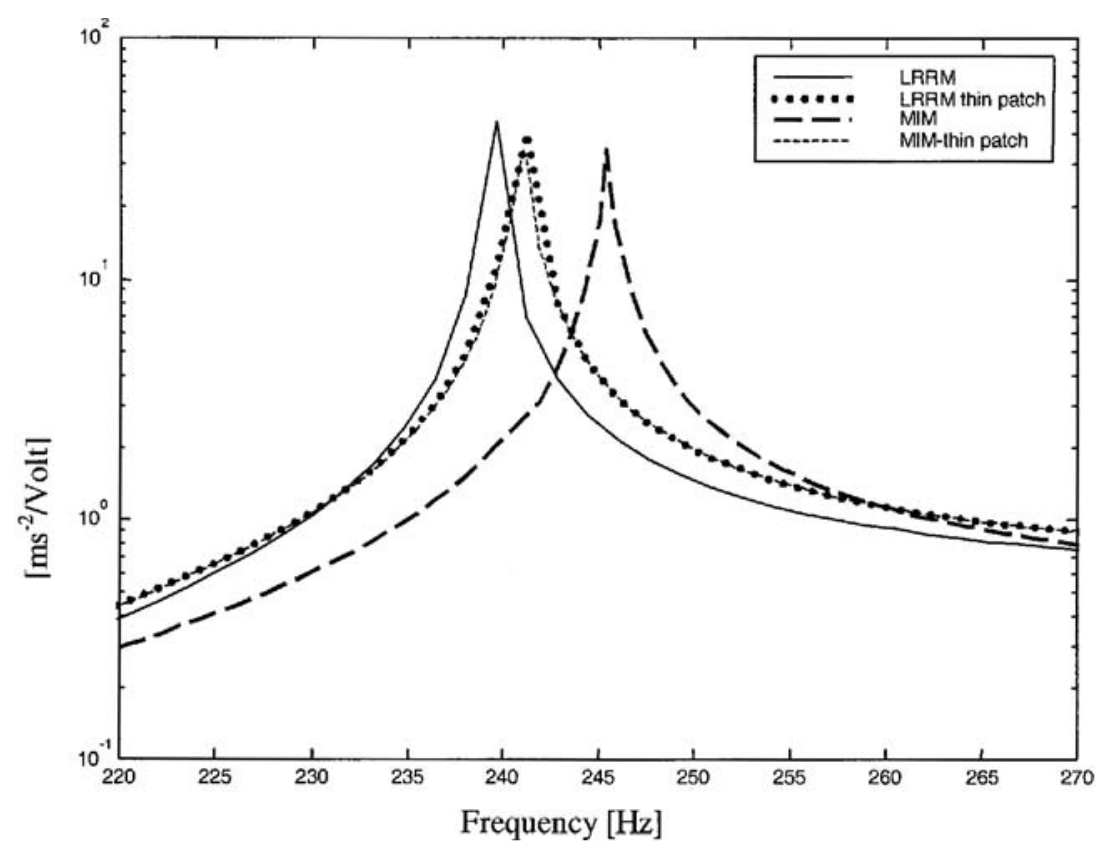

Fig. 12 Second resonance peak for normal and thin patches

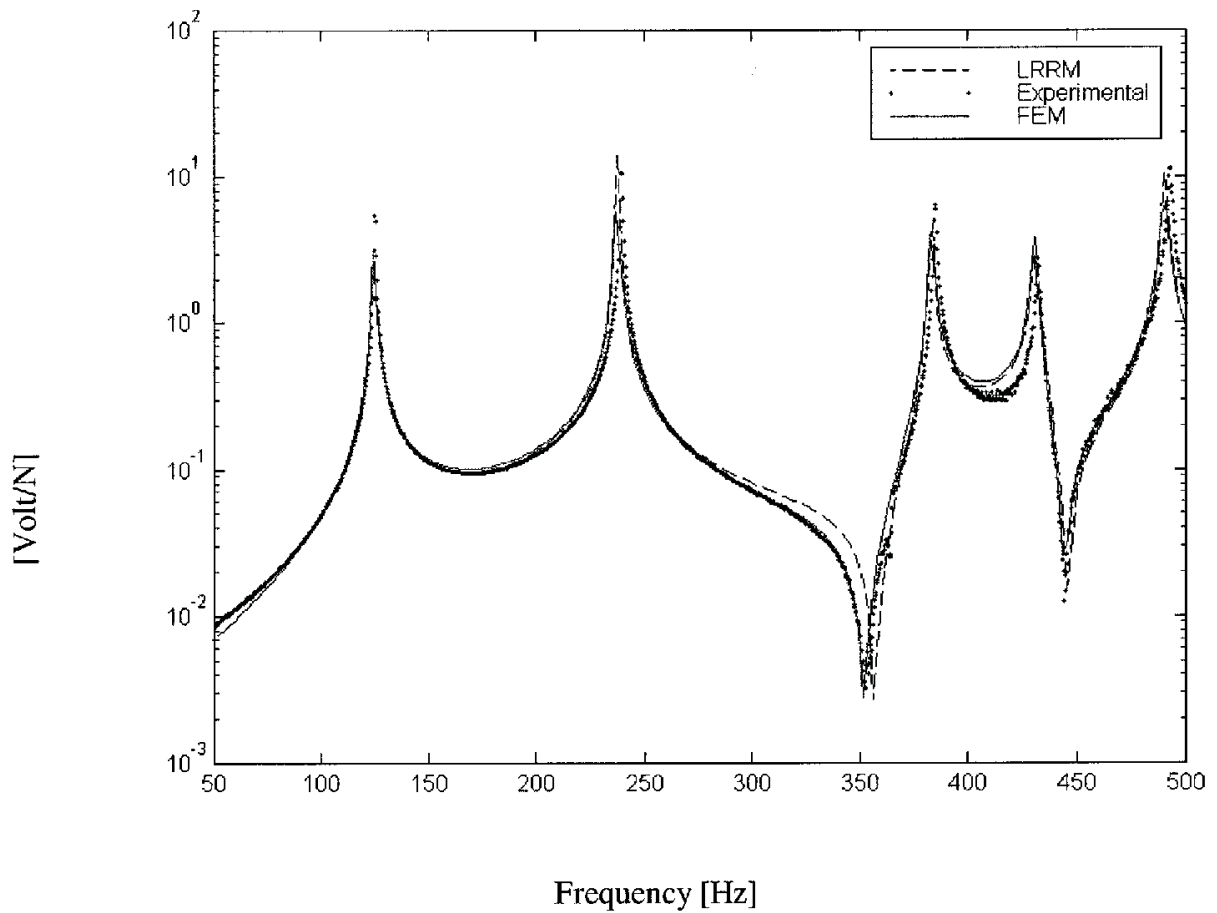

Fig. 13 Transfer function between applied force on the panel and voltage at the piezoelectric patch (sensor)

\section{CONCLUSIONS}

In this work, three techniques for the mathematical modelling of a piezoelectric actuated thin panel, namely the FE model, the LRR model and the MI model, have been briefly presented. An experimental implementation of a piezoelectric actuated simply supported panel, whose dynamics can be simulated using the mathe-

G00502 (C) IMechE 2002 matical models, is described in detail. The experimental results, i.e. transfer functions between the voltage applied to the piezoelectric patch and the acceleration at a specific location on the panel, are compared with the response predicted by the three mathematical models. The FE and LRR models are able to reproduce the dynamic behaviour of the panel with great accuracy, whereas the MI model even though able to capture the

Proc Instn Mech Engrs Vol 216 Part G： J Aerospace Engineering 
overall pattern of the response, was less accurate. The main reason for this is that the MI model neglects the out-of-plane inertia of the patch.

The capability of the LRR and FE models to simulate the dynamics of the system with the piezoelectric patch working as a sensor was also investigated. The transfer functions between a point force applied perpendicularly to the panel and the voltage produced at the electrodes of the patch were compared. The LRR model was able to reproduce accurately the experimental transfer function in the whole frequency range examined.

The verification of the models presented in this paper considered a simply supported panel, and therefore the validation is limited to this type of support. However, different boundary conditions may be implemented by using appropriated shape functions in the theoretical models (MI and LRR). Provided that these shape functions are adequate representations of the displacement field, the conclusions drawn in this paper should still be applicable to other types of supports.

\section{REFERENCES}

1 Fuller, C. R., Nelson P. A. and Elliott, S. J. Active control of vibrations, 1996, (Academic Press, London).

2 Garcia, E., Dosch, J. and Inman, D. J. The application of smart structures to the vibration suppression problem. J. Intelligent Material Systems Structures, 1992, 3.

3 Guigou, C., Fuller, R. C. and Wagstaff, P. R. Active isolation of vibration with adaptive structures. J. Acoustic Soc. Amer. 1994, 23(94), 294-299.

4 Jones, D. J., Prasad, S. E. and Wallace, J. B. Piezoelectric materials and their applications. Key Engineering Materials, 1996, 122-124.

5 Crawley, F. E. and De Luis, J. Use of piezoelectric actuators as elements of intelligent structures. AIAA J. 1987, 25(5).
6 Hagood, N., Chung, W. H. and Von Flotow, A. Modelling of piezoelectric actuator dynamics of active structural control. AIAA Paper 90-7087-CP, 1990.

7 Zhou, S.-W., Liang, C. and Rogers, C. A. Modelling of distributed piezoelectric actuators integrated with thin cylindrical shells. J. A coustic Soc. Amer. 1994, 96(3).

8 Lalande, F., Chaudhry, Z. and Rogers, C. A. Imped ancebased modelling of actuators bonded to shell structures. In Proceedings of the SPIE, Smart Structures and Materials 1995 Smart Structures and Integrated Systems, 2443, 1995, pp. 396-408.

9 Tzou, H. S. Neural sensation and actuation of shells using distributed piezoelectric transducers. In Proceedings of the 4th International Conference on Recent Advances in Structural Dynamics 1991, University of Southampton, ISVR, UK, pp. 668-678.

10 Banks, H. T., Smith, R. C. and Wang, Yun The modelling of piezoceramic patch interactions with shells, panels, and beams. Q. A ppl. Maths, 1995, LIII(2), 353-381.

11 Aglietti, G. S., Gabriel, S. B., Langley, R. S. and Rogers, E. A modelling technique for active control design studies with application to spacecraft microvibrations. J. Acoustic. Soc. Amer. 1997, 102(4) 2158-2166.

12 Zhou, S., Liang, C. and Rogers, C. A. A dynamic model of piezoelectric actuator-driven thin plate. In Proceedings of the SPIE, Smart Structures and Materials 1994, Smart Structures and Intelligent Systems, 1994, Vol. 2190.

13 Lefebvre, S., Guigou, C. and Fuller, C. R. Experiments on active isolation using distributed PVDF error sensor. $J$. Sound Vibration, 1992, 155, 177-184.

14 Aglietti, G. S. and Cunningham, P. R. Is a simple support really that simple? J. Sound Vibration, 2002, 257(2), 321335.

15 Timoshenko, S. Vibrational problems in engineering 4th Edition.

16 Data Acquisition Toolbox, Version 2, 1993 (Signal Processing Technology Ltd, Southampton, UK).

17 MATLAB 1997 (The Math Works Inc.).

18 Brennan, M. J., Day, M. J., Elliott, S. J. and Pinnington, R. J. Piezoelectric actuators and sensors. In Proceedings of the IUTAM Symposium on the Active Control of Vibrations, 1994. 